\title{
Efficiency of combined action of antimicrobial preparations against poly-resistant strains of conditionally-pathogenic bacteria isolated from wounds of surgery patients
}

\author{
T. V. Sklyar*, K. V. Lavrentieva*, O. M. Rudas*, O. V. Bilotserkivska*, \\ N. V. Kurahina*, M. G. Papiashvili**, O. A. Lykholat*** \\ * Oles Honchar Dnipro National University, Dnipro, Ukraine \\ **Independent laboratory Invitro LLC, Dnipro, Ukraine \\ ***University of Customs and Finance, Dnipro, Ukraine
}

Article info

Received 16.07.2020

Received in revised form 20.08.2020

Accepted 21.08.2020

Oles Honchar Dnipro National University,

Gagarina Ave., 72

Dnipro, 49010, Ukraine.

Tel.: +38-050-940-00-98.

E-mail:microviro@ukr.net

Independent laboratory

Invitro $L L C$,

Sviatoslav Khorobryi st., 38 ,

Dnipro, 49000, Ukraine.

Tel.: + 38-067-280-59-03.

E-mail:lena96dp@gmail.com

University of Customs

and Finance,

Volodymyr Vernadsky st., 2/4,

Dnipro, 49000, Ukraine.

$\mathrm{Tel} \cdot+38-066-038-68-92$

E-mail: lykholat2010@ukr.net

\section{Introduction}

The most relevant problem of contemporary medical practice is the development and spread of resistance of infectious agents to antibacterial preparations, leading to loss of clinical significance of a number of medical preparations due to absolute therapeutic inefficacy, significant losses of medical resources, increase in expense of treating patients and duration of their stay in medical institutions (Velez \& Sloand, 2016; Pervical et al., 2016; Rather et al., 2017; Aslam et al., 2018). According to recent data, 700 thousand patients die every year due to infections caused by antibiotic-resistant strains of microorganisms (Jenull et al., 2017; Tacconelli et al., 2018; Pachori et al., 2019).

In 2001, the WHO adopted and approved the fundamental document "Global strategy for containment of antimicrobial resistance" in which emergence and formation of antibiotic-resistant strains of microorganisms is recognized by representatives of countries of the EU and North America as a problem of international scale, and the main approaches to prevention of spread of resistant cultures were determined. In Febrary 2017, the WHO for the first time published a list of bacteria with high level of resistance to the action of antibiotics, which pose the greatest threat to the health of people. Out of this list, to the group of critically high level of antibiotic-resistance, such species of microorganisms were identified as Enterococcus faecium, Staphylococcus aureus, Klebsiella pneumoniae,
Acinetobacter baumannii, Pserc
species (Botelho et al., 2019).

Before the broad introduction of antibacterial preparations to medical practice, microorganisms were characterized by a high extent of natural susceptibility to antibiotics. Uncontrolled mass and prolonged use of the latter contributed to the gradual development and spread of resistant pathogenic and conditionally-pathogenic microorganisms in the environment, antibiotic-resistance of which is related not only to biochemical (modification of target of action, change in penetrability of cytoplasmic membrane of microbial cell, efflux, inactivation of active agent), but also to genetic mechanisms. Bacteria can become antibiotic-resistant as a result of mutations and horizontal transfer of genes localized in mobile genetic elements, genomic islands, phages, plasmids (Kramer et al., 2016; Von Wintersdorff et al., 2016). Furthermore, microorganisms may have not only interstrain and intraspecies, but also inter-genus transfer of antibioticresistance genes, which would contribute to emergence of cultures with obtained resistance to antibiotics and development of multidrug-resistant variants (Campbell et al., 2018; Cheng et al., 2019).

Until recently, the most effective preparations against poly-resistant strains of bacteria were considered to be aminoglycoside (gentamicin, amikacin, netilmicin), carbapenems (imipenem, meropenem), cephalosporins (ceftazidime, cefepime), fluoroquinolones (ciprofloxacin, levofloxacin), penicillins with $\beta$-Lactamase inhibitors (piperacillin / tazobac- 
tam), monobactams (aztreonam), phosphinous acids (fosfomycin) and polymyxins (colistin, polymyxin B) (Chellat et al., 2016; Bassetti et al., 2018; Marques et al., 2018). In recent years, there has been a significant increase in the resistance of clinical microbial isolates to preparations of, for example, fluoroquinolones and $\beta$-lactam antibiotics, imposing narrow limits on the therapeutic capabilities of contemporary medicine against agents of infectious diseases (Dalhoff, 2012; Agyekum et al., 2016; Anesi et al., 2016).

Apart from antibiotics, medical practice also applies antiseptics (Campbell et al., 2018; Palchykov et al., 2019; Zazharskyi et al., 2019). They are used in low concentrations for desinfection of skin and mucous membranes, surfaces of wounds. They include preparations of iodine, chlorine (halogen-containing substances), hydrogen peroxide (oxidant), miramistin, chlorhexidine (cationic detergents), calendula tincture, extract from eucalypt (preparations of plant origin), etc. (Williamson et al., 2017; Pachori et al., 2019). Antiseptics are prescribed mostly for external application (solutions of chlorhexidine bigluconate, hydrogen peroxide, ethyl alcohol, etc.), some preparations are used internally (furazidin, sodium hypochlorite and others) (Pachori et al., 2019).

Antiseptics are characterized by their broad range of action. They have no specific targets. Their antibacterial effect is associated with the processes of denaturalization of protein, damaging the penetrability of cytoplasmic membrane, inhibition of activity of enzymes of the microflora they affect on (Al-Talib et al., 2019). Recently, reports that constant use of antiseptics underlies increase in the number of strain pathogens resistant to them emerge more and more often (Matthew et al., 2017; Sweeney et al., 2018). At the same time, there are data that during their use in combination with other active substances, they, by contrast, increase the antibacterial effect of the latter (Williamson et al., 2017). Antiseptics are known to be capable of increasing susceptibility of antibiotic-resistant strains of microorganisms to penicillin, chloramphenicol, tetracycline, streptomycin, kanamycin, neomycin, novobiocin, erythromycin and cephalosporins, fluoroquinolones (Jenull et al., 2017; Pachori et al., 2019).

Regarding the relevance of the topic, the objective of the work was determining the efficiency of the combined effect of antimicrobial preparations on poly-resistant strains of conditionally pathogenic bacteria isolated from wounds of surgery patients.

\section{Materials and methods}

The study was performed on the basis of the Microbiology Department of PJC Nezalezhna Laboratoria Invitro (Dnipro) and the Department of Microbiology, Virulogy and Biotechnologies of the Dnipro National University. The objects of the study were clinical strains of conditionally pathogenic bacteria isolated from wounds of surgery patients: Staphylococcus aureus (8), S. epidermidis (10), Escherichia coli (14), Klebsiella pneumoniae (8), Proteus vulgaris (8), Proteus mirabilis (7), Pseudomonas aeruginosa (12).

Species of the isolated strains of enterobacteria and Pseudomonas aeruginosa were identified using test-kit API 20E (BioMerieux, France), staphylococci - using API Staph (BioMerieux, France).

Then, in the isolated cultures of conditionally pathogenic bacteria, we determined the extent of susceptibility to a number of antibiotic preparations of various pharmacological groups using disk diffusion method and method of minimum serial dilutions (ISO 20776-1.2006). For disk diffusion method we used standard disks with antibiotics (Hi Media, India). The spectrum of antibiotic preparations included: penicillins (ampicillin, oxacillin, amoxicillin/clavulanat acid), cephalosporins (cefotaxime, ceftriaxone, cefoxitin, cefuroxime, cefepime, ceftazidime), fluoroquinolones (ciprofloxacin, levofloxacin, ofloxacin, norfloxacin, gatifloxacin), aminoglycosides (amikacin, gentamicin, kanamycin, neomycin, netilmicin), tetracyclines (tetracycline), carbapenems (meropenem). The susceptibility of isolated bacterial strains to antibiotics was assessed according to the criteria CLSI/NCCLS, the extent of sensitivity was determined using the system SIR (EUCAST, 2015). As reference strains we used: $S$. aureus ATCC 29213, S. aureus ATCC 25923, E. coli ATCC 25922, P. aeruginosa ATCC 27853. Further, all the isolates, according to the generally accepted classification, were divided into phenotypic groups characterized by different extent of antibiotic-resistance (Magiorakos et al., 2012).
The reference method to determine susceptibility of isolated pathogens to antibiotics and antiseptics was the method of serial dilutions. It allows us also to determine minimum inhibitory concentration (MIC) of antibiotic / antiseptic or their combination against the tested strain. In the experiment we used two antiseptics most commonly applied in medical practice: decasan and chlorhexidine.

While using the methods of seial dilutions to prepare inocula, we used $24 \mathrm{~h}$ bacterial culture. Prior to inoculation to the medium, optical density of bacterial suspension of each test culture was adjusted to 0.5 according to the McFarland standard. From the main solutions of antibacterial preparations we prepared series of two-fold dillusions in test tubes to which we introduced the tested strains of microorganisms. At the same time, two controls were made. The inoculated test tubes were incubated in a thermostat at the temperature of $37^{\circ} \mathrm{C}$, during $18-24 \mathrm{~h}$, and then we monitored changes in the turbidity of the medium in the test ube. MIC of antibacterial preparations or their combinations were determined according to the concentration of antibacterial preparation or their combination in the last test tube where no growth of the tested strain was observed (Potapov et al., 2016).

\section{Results}

During the study of antibiotic-resistant clinical strains of conditionally pathogenic bacteria isolated from wounds of surgery patients, using disk diffusion method, we determined that the cultures were most resistant to antibiotics of the penicillin group: ampicillin $-89.6 \%$, oxacillin $-86.6 \%$, amoxicillin/clavulanat $-73.1 \%$ of the cultures. A high level of resistance remained also to tetracycline $-82.0 \%$ and cephalosporin of the II generation - cefoxitin $-70.2 \%$. To most antibiotics, the share of found resistant cultures was within $53.7-65.7 \%$. Greater effectiveness against the strains of conditionally pathogenic bacteria was exerted by preparations of last generations. Therefore, cephalosporins ceftazidime (III) and cefepime (IV) inhibited growth of $56.7 \%$ and $62.7 \%$, and fluoroquinolones levofloxacin and gatifloxacin (II) - respectively $58.2 \%$ and $62.7 \%$ of the isolates. The microbial cultures were found to be most susceptible to meropenem. Frequency of isolates resistant to this antibiotic did not exceed $35.8 \%$.

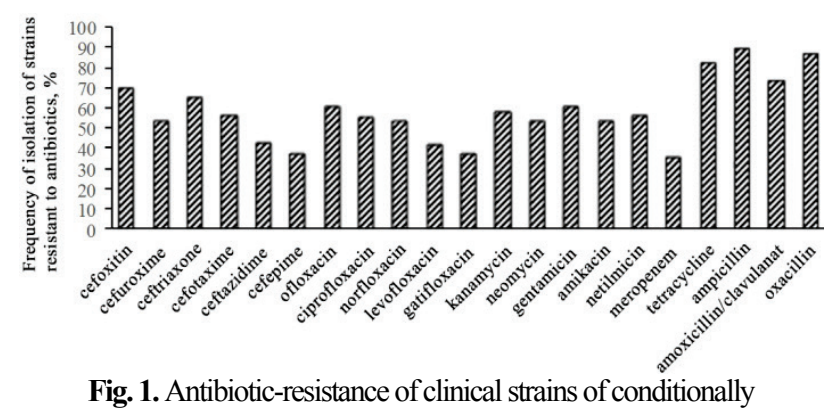

Fig. 1. Antibiotic-resistance of clinical strains of conditionally pathogenic bacteria isolated from wounds of surgery patients $(n=67)$

As with the intraspecies division of antibiotic-resistant isolates, $75.0 \%$ of strains of Staphylococcus aureus were characterized by resistance to cefoxitin, ceftriaxone, ofloxacin, tetracycline, ampicillin and $62.5 \%$ - to ciprofloxacin, oxacillin and most aminoglycosides (Table 1). $60.0 \%$ of clinical isolates of Staphylococcus epidermidis were resistant to cefoxitin, cefotaxime, ciprofloxacin, tetracycline and ampicillin. Regarding representatives of Enterobacteriaceae family, the least efficient were antibiotics tetracycline (to which resistance was exhibited by $71.4 \%$ of isolates of Escherichia coli, $75.5 \%$ of $K$. pneumoniae and all isolated cultures of Proteus) and penicillin preparations (the share of resistant tested isolates of enterobacteria exceeded $71.4 \%$ ). Moreover, $64.3 \%$ strains of E. coli were characterized by resistance to cefoxitin, cefotaxime, gentamicin and $71.4 \%$ to ceftriaxone; $62.5 \%$ of isolates of $K$. pneumoniae - to ceftriaxone, cefotaxime, norfloxacin and kanamycin and $75.0 \%$ - to cefoxitin; $62.5 \%$ of strains of P.vulgaris - to ceftriaxone, cefotaxime, ofloxacin, kanamycin and gentamicin; $71.4 \%$ of cultures of $P$. mirabilis - to cefoxitin and ofloxacin. The highest number of antibiotic-resistant isolates occurred among $P$. aeruginosa: $66.7 \%$ of the cultures were resistant to cefuroxime, ofloxacin and norfloxacin; $75.0 \%$ - to ceftriaxone and gentamicin; $83.3 \%$ aminoglycoside antibiotics; $91.7 \%$ - to cefoxitin. All isolated cultures of 
Pseudomonas aeruginosa were characterized by resistance to tetracycline and all tested penicillin preparations.

We determined that among antibiotic-resistant isolates, there occurred those which exhibited resistance to several antibiotics at the same time, thus, according to the classification generally accepted in medical practice, they were divided into corresponding phenotypic groups: MDR-strains multidrug-resistance - insensitive to at least one preparation of three or more classes; XDR - extensive drug-resistance - insensitive to at least one preparation in all the tested classes of antibiotics except one-two classes;
PDR - pandrug-resistance - insensitive to all antibiotics of all the tested classes. Among the isolated strains of $S$. aureus we found 4 poly-resistant isolates, of which two were identified to MDR- and one of each XDRand PDR-groups. Pandrug-resistant strain 1 was characterized by resistance to all antibacterial preparations except cefepime, extensive drugresistant strain 4 - to tetracycline, gentamicin, ofloxacin, ceftriaxone, ampicillin; polyresistant strain 5 - tetracycline, ceftriaxone, oxacillin and gentamicin; poly-resistant strain 7 - ofloxacin, gentamicin and ampicillin (Table 2).

\section{Table 1}

Frequency of isolation of antibiotic-resistant isolates from the contents of wounds of surgery patients (absolute number of $/ \%$ of the number of strains within species)

\begin{tabular}{|c|c|c|c|c|c|c|c|}
\hline Antibiotic & S. aureus, $\mathrm{n}=8$ & S. epidermidis, $\mathrm{n}=10$ & E. coli, $\mathrm{n}=14$ & K. pneumoniae, $\mathrm{n}=8$ & P. vulgaris, $\mathrm{n}=8$ & P. mirabilis, $\mathrm{n}=7$ & P. aeruginos $a, \mathrm{n}=12$ \\
\hline Cefoxitin & $6 / 75.0$ & $6 / 60.0$ & $9 / 64.3$ & $6 / 75.0$ & $4 / 50.0$ & $5 / 71.4$ & $11 / 91.7$ \\
\hline Cefuroxime & $4 / 50.0$ & $5 / 50.0$ & $8 / 57.1$ & $3 / 37.5$ & $4 / 50.0$ & $4 / 57.1$ & $8 / 66.7$ \\
\hline Ceftriaxone & $6 / 75.0$ & $5 / 50.0$ & 10/71.4 & $5 / 62.5$ & $5 / 62.5$ & $4 / 57.1$ & $9 / 75.0$ \\
\hline Cefotaxime & $4 / 50.0$ & $6 / 60.0$ & $9 / 64.3$ & $5 / 62.5$ & $5 / 62.5$ & $2 / 28.6$ & $7 / 58.3$ \\
\hline Ceftazidime & $3 / 37.5$ & $4 / 40.0$ & $7 / 50.0$ & $3 / 37.5$ & $4 / 50.0$ & $3 / 42.9$ & $5 / 41.7$ \\
\hline Cefepime & $2 / 25.0$ & $3 / 30.0$ & $6 / 42.9$ & $2 / 25.0$ & $3 / 37.5$ & $3 / 42.9$ & $6 / 50.0$ \\
\hline Ofloxacin & $6 / 75.0$ & $5 / 50.0$ & $8 / 57.1$ & $4 / 50.0$ & $5 / 62.5$ & $5 / 71.4$ & $8 / 66.7$ \\
\hline Ciprofloxacin & $5 / 62.5$ & $6 / 60.0$ & $7 / 50.0$ & $4 / 50.0$ & $4 / 50.0$ & $4 / 57.1$ & $7 / 58.3$ \\
\hline Norfloxacin & $4 / 50.0$ & $5 / 50.0$ & $6 / 42.9$ & $5 / 62.5$ & $4 / 50.0$ & $4 / 57.1$ & $8 / 66.7$ \\
\hline Levofloxacin & $3 / 37.5$ & $5 / 50.0$ & $5 / 35.7$ & $4 / 50.0$ & $3 / 37.5$ & $2 / 28.6$ & $6 / 50.0$ \\
\hline Gatifloxacin & $2 / 25.0$ & $4 / 40.0$ & $4 / 28.6$ & $3 / 37.5$ & $3 / 37.5$ & $3 / 42.9$ & $6 / 50.0$ \\
\hline Kanamycin & $4 / 50.0$ & $5 / 50.0$ & $6 / 42.9$ & $5 / 62.5$ & $5 / 62.5$ & $4 / 57.1$ & $10 / 83.3$ \\
\hline Neomycin & $3 / 37.5$ & $3 / 30.0$ & $8 / 57.1$ & $4 / 50.0$ & $4 / 50.0$ & $4 / 57.1$ & $10 / 83.3$ \\
\hline Gentamicin & $5 / 62.5$ & $5 / 50.0$ & $9 / 64.3$ & $4 / 50.0$ & $5 / 62.5$ & $4 / 57.1$ & $9 / 75.0$ \\
\hline Amikacin & $5 / 62.5$ & $5 / 50.0$ & $6 / 42.9$ & $4 / 50.0$ & $3 / 37.5$ & $3 / 42.9$ & $10 / 83.3$ \\
\hline Netilmicin & $5 / 62.5$ & $4 / 40.0$ & $7 / 50.0$ & $4 / 50.0$ & $4 / 50.0$ & $4 / 57.1$ & $10 / 83.3$ \\
\hline Meropenem & $2 / 25.0$ & $4 / 40.0$ & $5 / 35.7$ & $2 / 25.0$ & $3 / 37.5$ & $3 / 42.9$ & $5 / 41.7$ \\
\hline Tetracycline & $6 / 75.0$ & $6 / 60.0$ & $10 / 71.4$ & $6 / 75.0$ & $8 / 100.0$ & $7 / 100.0$ & $12 / 100.0$ \\
\hline Ampicillin & $6 / 75.0$ & $6 / 60.0$ & $13 / 92.9$ & $8 / 100.0$ & $8 / 100.0$ & $7 / 100.0$ & $12 / 100.0$ \\
\hline Amoxicillin/clavulanat & $4 / 50.0$ & $3 / 30.0$ & $11 / 78.6$ & $7 / 87.5$ & $6 / 75.0$ & $6 / 85.7$ & $12 / 100.0$ \\
\hline Oxacillin & $5 / 62.5$ & $5 / 50.0$ & $13 / 92.9$ & $8 / 100.0$ & $8 / 100.0$ & $7 / 100.0$ & $12 / 100.0$ \\
\hline
\end{tabular}

Among the isolated strains of $S$. epidermidis, we found no pandrugresistant ones. Only one strain 3, resistant to ofloxacin, tetracycline, ceftriaxone, cefoxitin, gentamicin and ampicillin, was identified to phenotype XDR; another one -7 to MDR, with resistance to ceftriaxone, ofloxacin and gentamicin. Pandrug-resistant isolates were not found also among strains of K. pneumoniae. Two isolates -4 and 7, which exhibited high resistance to such antibacterial preparations as ceftriaxone, gentamicin, ofloxacin, oxacillin and ampicillin, were identified to MDR-strains, and strain 6, which was resistant to gentamicin, ceftriaxone, ofloxacin, tetracycline and ampicillin, - to XDR.

\section{Table 2}

Classification to phenotypic groups

of antibiotic-resistant isolates from wounds of surgery patients

\begin{tabular}{lccc}
\hline \multirow{2}{*}{ Strain } & \multicolumn{3}{c}{ Number of strains in phenotype group } \\
\cline { 2 - 4 } & MDR & XDR & PDR \\
\hline S. aureus, $\mathrm{n}=8$ & 2 & 1 & 1 \\
S. epidermidis, $\mathrm{n}=10$ & 1 & 1 & 0 \\
E. coli, $\mathrm{n}=14$ & 3 & 2 & 1 \\
K.pneumoniae, $\mathrm{n}=8$ & 2 & 1 & 0 \\
P. vulgaris, $\mathrm{n}=8$ & 1 & 1 & 1 \\
P. mirabilis, $\mathrm{n}=7$ & 2 & 1 & 0 \\
P. aeruginos, $\mathrm{n}=12$ & 3 & 1 & 1 \\
\hline
\end{tabular}

According to the obtained results presented in Table 2, among eight isolated cultures of $P$. vulgaris, one pandrug-, one extensive drug- and one multidrug-resistant isolate was detetected. PDR-strain 1 was resistant to all antibacterial preparations except cefepime. Extensive drug-resistant strain 6 exerted resistance to ceftriaxone, gentamicin, tetracycline, ofloxacin, oxacillin and amoxicillin/clavulanat; multi-resistant strain 8 - to ceftriaxone, ofloxacin, gentamicin and ampicillin. Among eight isolated cultures of $P$. mirabilis, we determined one extensive drug- and two multidrugresistant strains. Extensive drug-resistant strain 3 demonstrated resistance to ceftriaxone, ofloxacin, gentamicin, tetracycline, meropenem, ampicillin. Multidrug-resistant strains 1 and 7 were characterized by resistance to ceftriaxone, ofloxacin, gentamicin. The highest number of poly-resistant isolates was found among species of $E$. coli and $P$. aeruginosa: three of
MDR-, two XDR- and one PDR-strains for each of the species. Among isolates of E. coli, PDR-strain 2 was resistant to all the antibiotics except levofloxacin; XDR - 6 - to ceftriaxone, ofloxacin, gentamicin, tetracycline, ampicillin; XDR - 1 - ceftriaxone, ofloxacin, tetracycline, gentamicin, amoxicillin/clavulanat. MDR-strains of E. coli 3 and 10 were resistant to ceftriaxone, ofloxacin, gentamicin, and strain 13 - also to tetracycline. Among the isolated cultures of $P$. aeruginos $a$, pandrug-resistant strain 9 was characterized by resistance to all the tested antibiotics except ceftazidime, and extensive drug-resistant -6 - to ceftriaxone, gentamicin, meropenem, tetracycline, ofloxacin and oxacillin. Multiresistant strains 2 and 8 exhibited high resistance to ceftriaxone, gentamicin and ofloxacin; and strain 12 - also to ciprofloxacin.

The results we obtained demonstrate that most poly-resistant clinical strains of conditionally pathogenic bacteria isolated from wounds of surgery patients were characterized by resistance to three antibiotics: ceftriaxone, ofloxacin and gentamicin. Therefore, in the next stage of the survey we determined the minimum inhibitory concentration (MIC) of particularly these antibacterial preparations against resistant-to-them PDR-, XDRMDR-isolates (Table 3). For MDR-strains of Staphylococcus aureus (5 and 7), the values of MIC of ceftriaxone, ofloxacin and gentamicin coincided with the threshold values (respectively 64,8 and $16 \mu \mathrm{g} / \mathrm{mL}$ ), for XDR-strain 4 - twice exceeded the threshold values, and for PDR-strain 1 - exceeded the values by four times. As with antibiotic-resistant isolates of Staphylococcus epidermidis, for MDR-strain 7, the MIC of ceftriaxone corresponded to the threshold value, and MIC of ofloxacin and gentamicin - exceeded it two-fold. For XDR-strain 3 of Staphylococcus epidermidis, the experimental values of MIC of three tested antibiotics exceeded the corresponding threshold value by 4 times.

MIC values for ceftriaxone, ofloxacin and gentamicin for MDRstrain 4 of $K$. pneumoniae exceeded the normative value by 2 and 4 times; and for MDR-strain 7 coinsided with them. The extremely-resistant strain $K$. pneumoniae 6 showed the high level of resistance, therefore values of MIC of all three tested antibiotics for this isolate were higher than the norm values by 8,4 and 2 times. PDR-strain of $P$. vulgaris 1 was found to be highly resistant. MIC of ceftriaxone for this isolate equaled $256.0 \mu \mathrm{g} / \mathrm{mL}$ with the norm $\geq 64.0 \mu \mathrm{g} / \mathrm{mL}$, MIC of ofloxacin and gentamicin -64.0 
$\mu \mathrm{g} / \mathrm{mL}$, exceeding the norm parameters respectively by 8 and 4 times. Values of MIC of ceftriaxone and ofloxacin for XDR-strain 6 exceeded the threshold values by 2 and 4 times, MIC of gentamicin coincided with the normative value. For MDR-strain 7, the MIC values of ceftriaxone and gentamicin did not exceed the threshold value, MIC of ofloxacin was $16.0 \mu \mathrm{g} / \mathrm{mL}$, while the norm equals $8.0 \mu \mathrm{g} / \mathrm{mL}$. Extensive drug-resistant strain 3 of $P$. mirabilis was characterized by high level of resistance to three tested antibiotics. Against this strain, MIC of ceftriaxone equaled $256.0 \mu \mathrm{g} / \mathrm{mL}$, ofloxacin $-32.0 \mu \mathrm{g} / \mathrm{mL}$, gentamicin $-64.0 \mu \mathrm{g} / \mathrm{mL}$. As with multidrug-resistant isolates, exceess of MIC value of ceftriaxone was recorded only in relation to MDR-isolate 7, and that of ofloxacin and gentamicinfor MDR-isolate 7. For MDR-strains of E. coli (3, 10 and 13), the MIC values of ceftriaxone, gentamicin and ofloxacin coincided with the threshold values, for XDR-strains 6 and 11 - exceeded threshold values respectively by 2 and 4 times, and for PDR-strain 2 -by 4,2 and 8 times.

For MDR-strains of P. aeruginosa (12, 8 and 3), the MIC values of ceftriaxone coincided with the threshold values, and in the case of gentamicin and ofloxacin were even lower than them. MIC value of gentamicin and ofloxacin against pan-resistant and extra-resistant isolates of $P$. aeruginosa were also lower than the threshold values of MIC of these antibiotics, by contrast to MIC of ceftriaxone: against XDR-strain 6, this parameter equaled $128.0 \mu \mathrm{g} / \mathrm{mL}$, and for PDR-isolate 9 - even higher, $256.0 \mu \mathrm{g} / \mathrm{mL}$

Table 3

Experimental values of minimum inhibitory concentration of antibiotics and antiseptics against poly-resistant isolates of conditionally pathogenic bacteria isolated from wounds of surgery patients

\begin{tabular}{|c|c|c|c|c|c|c|}
\hline \multirow{2}{*}{\multicolumn{2}{|c|}{ Strains }} & \multicolumn{3}{|c|}{ MIC of antibiotic, $\mu \mathrm{g} / \mathrm{mL}$} & \multicolumn{2}{|c|}{ MIC of antiseptic, $\mu \mathrm{g} / \mathrm{mL}$} \\
\hline & & ceftriaxone & ofloxacin & gentamicin & decasan & chlorhexidine \\
\hline \multirow{4}{*}{ S. aureus } & 1 (PDR) & 256.0 & 32.0 & 64.0 & 3.0 & 125.0 \\
\hline & 4 (XDR) & 128.0 & 16.0 & 32.0 & 100.0 & 125.0 \\
\hline & 5 (MDR) & 64.0 & 8.0 & 16.0 & 6.3 & 8.0 \\
\hline & 7 (MDR) & 64.0 & 8.0 & 16.0 & 100.0 & 62.5 \\
\hline \multirow{2}{*}{ S. epidermidis } & 3 (XDR) & 256.0 & 32.0 & 64.0 & 100.0 & 31.3 \\
\hline & 7 (MDR) & 64.0 & 16.0 & 32.0 & 3.0 & 31.3 \\
\hline \multirow{3}{*}{ K. pneumoniae } & 6 (XDR) & 256.0 & 64.0 & 32.0 & 3.0 & 16.0 \\
\hline & 4 (MDR) & 128.0 & 32.0 & 64.0 & 50.8 & 8.0 \\
\hline & 7 (MDR) & 64.0 & 8.0 & 16.0 & 3.0 & 31.3 \\
\hline \multirow{3}{*}{ P. vulgaris } & 1 (PDR) & 256.0 & 64.0 & 64.0 & 100.0 & 62.5 \\
\hline & 6 (XDR) & 128.0 & 32.0 & 32.0 & 3.0 & 250.0 \\
\hline & 8 (MDR) & 64.0 & 16.0 & 16.0 & 6.3 & 125.0 \\
\hline \multirow{3}{*}{ P. mirabilis } & 3 (XDR) & 256.0 & 32.0 & 64.0 & 100.0 & 125.0 \\
\hline & 1 (MDR) & 64.0 & 16.0 & 32.0 & 100.0 & 16.0 \\
\hline & 7 (MDR) & 128.0 & 8.0 & 16.0 & 6.3 & 250.0 \\
\hline \multirow{6}{*}{ E. coli } & 2 (PDR) & 256.0 & 64.0 & 32.0 & 6.3 & 125.0 \\
\hline & 6 (XDR) & 128.0 & 32.0 & 32.0 & 12.5 & 62.5 \\
\hline & 11 (XDR) & 128.0 & 32.0 & 32.0 & 6.3 & 4.0 \\
\hline & 3 (MDR) & 64.0 & 8.0 & 16.0 & 6.3 & 8.0 \\
\hline & 10 (MDR) & 64.0 & 8.0 & 16.0 & 12.5 & 250.0 \\
\hline & 13 (MDR) & 64.0 & 16.0 & 16.0 & 100.0 & 125.0 \\
\hline \multirow{5}{*}{ P. aeruginosa } & 9 (PDR) & 256.0 & 4.0 & 8.0 & 50.0 & 125.0 \\
\hline & 6 (XDR) & 128.0 & 4.0 & 4.0 & 25.0 & 125.0 \\
\hline & 3 (MDR) & 64.0 & 1.0 & 1.0 & 25.0 & 62.5 \\
\hline & 8 (MDR) & 64.0 & 2.0 & 2.0 & 12.5 & 31.3 \\
\hline & 12 (MDR) & 64.0 & 2.0 & 2.0 & 25.0 & 250.0 \\
\hline
\end{tabular}

Thus, the obtained results demonstrate that significant increase in the threshold values of MIC of the tested antibiotics were recorded against pandrug-resistant and extensive drug-resistant clinical isolates of bacteria from contents of wounds of surgery patients.

In the next stage of work the subject of interest was the experimental determining of MIC of decasan and chlorhexidine against poly-resistant isolates of the tested cultures of microorganisms (Table 3). Out of four tested strains of Staphylococcus aureus, the most resistant to the action of both antibiotics were XDR-strain 4 and MDR-strain 5. MIC of decasan and chlorhexidine against these isolates reached quite high values: respectively $100.0,125.0$ and $62.5 \mu \mathrm{g} / \mathrm{mL}$. Among the isolates, the most susceptible to decasan was pan-resistant isolate 1, against which MIC of decasan exceeded 3.0; at the same time, MIC of chlorhexidine was high $125.0 \mu \mathrm{g} / \mathrm{mL}$. Decasan- and chlorhexidine-susceptibility was recorded for MDR-strain 5, in this case MIC of two antiseptics respectively equaled 6.3 and $8.0 \mu \mathrm{g} / \mathrm{mL}$. MDR-strain 7 of $S$. epidermidis was also susceptible to decasan and chlorhexidine. Against this strain, MIC of decasan was $3.0 \mu \mathrm{g} / \mathrm{mL}$, and that of chlorhexidine $-31.3 \mu \mathrm{g} / \mathrm{mL}$. XDR-strain 3 was characterized by high level of resistance to antiseptics: MIC of chlorhexidine $=31.3 \mu \mathrm{g} / \mathrm{mL}$, and MIC of decasan equaled even more $100.0 \mu \mathrm{g} / \mathrm{mL}$. Decasan was found to be an effective antiseptic also against poly-resistant strains of Klebsiella. Therefore, against MDR-strain 7 and XDR-strain 6, MIC of decasan $=3.0 \mu \mathrm{g} / \mathrm{mL}$, unlike MDR-strain 4, for which MIC of decasan reached the value of $50.8 \mu \mathrm{g} / \mathrm{mL}$. However, opposite results were obtained regarding minimum inhibitory concentration of decasan and chlorhexidine, equaling respectively 31.3, 16.0 and $8.0 \mu \mathrm{g} / \mathrm{mL}$ against tested poly-resistant strains of $K$. pneumoniae. High bactericidal activity was exhibited by decasan towards XDR-isolate 6 ( $\mathrm{MIC}=3.0 \mu \mathrm{g} / \mathrm{mL}$ ), MDR-isolate 8 of $P$. vulgaris and MDR-strain $7 \mathrm{of}$ P. mirabilis $(\mathrm{MIK}=6.3 \mu \mathrm{g} / \mathrm{mL})$. It had low activity against other tested isolates of Proteus (MIC $=100.0 \mu \mathrm{g} / \mathrm{mL}$ ). The highest resistance to chlorhexidine was observed for XDR-isolate 6 of $P$. vulgaris and MDR-isolate 1 of $P$. mirabilis. MIC of chlorhexidine against these isolates reached the value of $250.0 \mu \mathrm{g} / \mathrm{mL}$. Somewhat lower $(125.0 \mu \mathrm{g} / \mathrm{mL})$ MIC of chlorhexidine was seen for MDR-strain 8 of $P$. vulgaris and XDR-strain 3 of $P$. mirabilis. The most sensitive isolate to the action of chlorhexidine was MDR-strain 1 of $P$. mirabilis. In this case MIC of chlorhexidine did not exceed $16.0 \mu \mathrm{g} / \mathrm{mL}$. Practically all the poly-resistant isolates of Escherichia coli were susceptible to decasan except MDR-strain 13. Against it, MIC of decasan equaled $100.0 \mu \mathrm{g} / \mathrm{mL}$. As with chlorhexidine, the lowest value of its MIC was determined only against two isolates of $E$. coli: XDR 11 and MDR 3. Other poly-resistant strains of $E$. coli were quite resistant to this antiseptic. Its MIC against these cultures exceeded the value of $62.5 \mu \mathrm{g} / \mathrm{mL}$. High susceptibility to decasan was seen in all the tested isolates of Pseudomonas aeruginosa. Its MIC was within 12.5 to $50.0 \mu \mathrm{g} / \mathrm{mL}$. All the cultures were resistant to chlorhexidine, as demonstrated by MIC of the preparation equaling higher than $31.3 \mu \mathrm{g} / \mathrm{mL}$.

The final stage of our study was research on the combined effect of chlorhexidine and decasan with ceftriaxone, ofloxacin and gentamicin against the tested bacterial strains. Accoding to the data of Table 4 and comparison of them to the data of Table 3 , we can draw a conclusion that towards a larger amount of the studied cultures the bactericidal effect of antibiotics increased during their combined application with antiseptics, manifesting through decrease in MIC of the tested preparations. 
The greatest decrease in MIC of antibiotics to its threshold values, below which the culture could be considered susceptible, was seen while using combination gentamicin + decasan or chlorhexidine against all polyresistant isolates of Pseudomonas aeruginosa, strains of E. coli 10 and 13, P. mirabilis 7, K. pneumoniae 7, S. aureus 5 and 7; ofloxacin + decasan or chlorhexidine against isolates of $P$. aeruginosa $6,3,8$ and 12 , P. mirabilis 7, K. pneumoniae 7, strains of $S$. aureus 5 and 7 ; ceftriaxone + decasan or chlorhexidine against strains of $P$. aeruginosa $3,8,12$ and $S$. aureus 7 . The greatest susceptibility to the combined action was observed in MDR-isolates of $P$. aeruginosa and $S$. aureus, the greatest resistance was shown by strains of $S$. epidermidis, $P$. vulgaris and E. coli.

During combined application of antiseptics and antibiotics, we observed a certain decrease in MIC against bacterial cultures, though not as actively as in the case of antibiotic preparations (mostly twofold). Insignificant decrease in MIC was seen for decasan and its combination with gentamicin against strain 6 and ceftriaxone, ofloxacin or gentamicinom against strain 7 of $K$. pneumoniae (by 0.75 times), and also ceftriaxone, ofloxacin or gentamicin against strains of $P$. vulgaris $8, P$. mirabilis 7 , E. coli 2, 3, 11 (by 1.5 times); chlorhexidine combined with ceftriaxone, ofloxacin and gentamicin against strains of $K$. pneumoniae 4,7 . It should be noted that in the following experimental variants: decasan + ceftriaxone, ofloxacin or gentamicin against strains of $S$. aureus 7, P. mirabilis 1 , E. coli $13, P$. aeruginosa 9 , and also chlorhexidine + ceftriaxone, ofloxacin or gentamicin against strains of $S$. aureus $7, P$. vulgaris 8, E. coli 2 , $P$. aeruginosa 3 and 8 , the value of MIC decreased to the threshold value, making the tested cultures susceptible to the effect of antiseptics.

\section{Table 4}

Pattern of combined effect of antibiotics and antiseptics on strains of poly-resistant bacteria isolated from wounds of surgery patients

\begin{tabular}{|c|c|c|c|c|c|c|c|}
\hline & \multirow[t]{2}{*}{ Strains } & \multicolumn{6}{|c|}{ MIC of antibiotic/antiseptic, $\mu \mathrm{g} / \mathrm{mL}$} \\
\hline & & $\mathrm{CF} / \mathrm{DC}$ & $\mathrm{OF} / \mathrm{DC}$ & $\mathrm{GM} / \mathrm{DC}$ & $\mathrm{CF} / \mathrm{CH}$ & $\mathrm{OF} / \mathrm{CH}$ & $\mathrm{GM} / \mathrm{CH}$ \\
\hline \multirow{4}{*}{ S. aureus } & 1 (PDR) & $128.0 / 1.5$ & $16.0 / 1.5$ & $32.0 / 1.5$ & $128.0 / 62.5$ & $16.0 / 62.5$ & $32.0 / 62.5$ \\
\hline & 4 (XDR) & $32.0 / 50.0$ & $8.0 / 50.0$ & $16.0 / 50.0$ & $32.0 / 62.5$ & $8.0 / 62.5$ & $16.0 / 31.3$ \\
\hline & 5 (MDR) & $16.0 / 1.5$ & $2.0 / 3.1$ & $4.0 / 3.1$ & $16.0 / 2.0$ & $2.0 / 4.0$ & $2.0 / 2.0$ \\
\hline & 7 (MDR) & $8.0 / 25.0$ & $2.0 / 25.0$ & $4.0 / 25.0$ & $16.0 / 15.7$ & $1.0 / 15.6$ & $2.0 / 15.6$ \\
\hline \multirow{2}{*}{ S. epidermidis } & 3 (XDR) & $128.0 / 50.0$ & $16.0 / 50.0$ & $32.0 / 50.0$ & $128.0 / 15.7$ & $16.0 / 15.6$ & $32.0 / 15.6$ \\
\hline & 7 (MDR) & $16.0 / 1.5$ & $8.0 / 1.5$ & $8.0 / 0.8$ & $16.0 / 7.8$ & $4.0 / 7.8$ & $8.0 / 15.6$ \\
\hline \multirow{3}{*}{ K. pneumoniae } & 6 (XDR) & $64.0 / 1.5$ & $32.0 / 1.5$ & $8.0 / 4.0$ & $64.0 / 4.0$ & $8.0 / 2.0$ & $8.0 / 4.0$ \\
\hline & 4 (MDR) & $32.0 / 25.0$ & $8.0 / 12.5$ & $8.0 / 25.0$ & $64.0 / 8.0$ & $32.0 / 8.0$ & $8.0 / 4.0$ \\
\hline & 7 (MDR) & $32.0 / 4.0$ & $4.0 / 4.0$ & $8.0 / 4.0$ & $16.0 / 31.3$ & $2.0 / 31.3$ & $4.0 / 31.3$ \\
\hline \multirow{3}{*}{ P. vulgaris } & 1 (PDR) & $64.0 / 25.0$ & $16.0 / 25.0$ & $16.0 / 25.0$ & $64.0 / 16.0$ & $32.0 / 31.3$ & $32.0 / 31.3$ \\
\hline & 6 (XDR) & $32.0 / 1.5$ & $16.0 / 1.5$ & $16.0 / 1.5$ & $32.0 / 125.0$ & $8.0 / 62.5$ & $8.0 / 125.0$ \\
\hline & 8 (MDR) & $32.0 / 4.0$ & $8.0 / 4.0$ & $8.0 / 4.0$ & $16.0 / 31.3$ & $8.0 / 62.5$ & $8.0 / 31.3$ \\
\hline \multirow{3}{*}{ P. mirabilis } & 3 (XDR) & $64.0 / 25.0$ & $4.0 / 25.0$ & $16.0 / 25.0$ & $64.0 / 62.5$ & $8.0 / 62.5$ & $32.0 / 62.5$ \\
\hline & 1 (MDR) & $16.0 / 25.0$ & $8,0 / 25.0$ & $8.0 / 25.0$ & $32.0 / 8.0$ & $8.0 / 8.0$ & $16.0 / 8.0$ \\
\hline & 7 (MDR) & $64.0 / 4.0$ & $4.0 / 4.0$ & $8.0 / 4.0$ & $32.0 / 62.5$ & $2.0 / 62.5$ & $4.0 / 62.5$ \\
\hline \multirow{6}{*}{ E. coli } & 2 (PDR) & $64.0 / 4.0$ & $32.0 / 4.0$ & $32.0 / 4.0$ & $32.0 / 31.3$ & $16.0 / 62.5$ & $32.0 / 31.3$ \\
\hline & $6(\mathrm{XDR})$ & $32.0 / 6.3$ & $16.0 / 6.3$ & $32.0 / 6.3$ & $32.0 / 31.3$ & $8.0 / 16.0$ & $16.0 / 31.3$ \\
\hline & 11 (XDR) & $32.0 / 4.0$ & $16.0 / 4.0$ & $16.0 / 4.0$ & $32.0 / 2.0$ & $16.0 / 2.0$ & $32.0 / 2.0$ \\
\hline & 3 (MDR) & $32.0 / 4.0$ & $4.0 / 4.0$ & $8.0 / 4.0$ & $32.0 / 4.0$ & $4.0 / 4.0$ & $8.0 / 2.0$ \\
\hline & 10 (MDR) & $32.0 / 6.3$ & $4.0 / 6.3$ & $4.0 / 6.3$ & $32.0 / 125.0$ & $4.0 / 125.0$ & $4.0 / 125.0$ \\
\hline & 13 (MDR) & $16.0 / 25.0$ & $4.0 / 50.0$ & $2.0 / 25.0$ & $16.0 / 62.5$ & $8.0 / 62.5$ & $4.0 / 31.3$ \\
\hline \multirow{5}{*}{ P. aeruginosa } & 9 (PDR) & $64.0 / 25.0$ & $4.0 / 25.0$ & $2.0 / 50.0$ & $64.0 / 62.5$ & $4.0 / 62.5$ & $2.0 / 62.5$ \\
\hline & 6 (XDR) & $32.0 / 12.5$ & $1.0 / 12.5$ & $1.0 / 12.5$ & $32.0 / 62.5$ & $1.0 / 62.5$ & $1.0 / 62.5$ \\
\hline & 3 (MDR) & $8.0 / 6.3$ & $0.5 / 6.3$ & $0.5 / 6.3$ & $8.0 / 3.3$ & $0.5 / 31.3$ & $0.5 / 62.5$ \\
\hline & 8 (MDR) & $16.0 / 6.3$ & $0.5 / 6.3$ & $0.5 / 3.1$ & $8.0 / 7.8$ & $0.5 / 3.9$ & $0.5 / 7.8$ \\
\hline & 12 (MDR) & $8.0 / 6.3$ & $0.5 / 3.1$ & $0.5 / 3.1$ & $8.0 / 62.5$ & $0.5 / 62.5$ & $0.5 / 62.5$ \\
\hline
\end{tabular}

Note: $\mathrm{CF} / \mathrm{DC}$ - combination of ceftriaxone and decasan; $\mathrm{OF} / \mathrm{DC}$ - combination of ofloxacin and decasan; $\mathrm{GM} / \mathrm{DC}$ - combination of gentamicin and decasan; $\mathrm{CF} / \mathrm{CH}-\mathrm{combi}-$ nation of ceftriaxone and chlorhexidine; $\mathrm{OF} / \mathrm{CH}$ - combination of ofloxacin and chlorhexidine; $\mathrm{GM} / \mathrm{CH}$ - combination of gentamicin and chlorhexidine.

\section{Discussion}

Currently, resistance of conditionally pathogenic strains of bacteria to antibacterial preparations is one of the main global problems in the sphere of healthcare. Its solution requires a complex approach. The strategy of use of combined therapy with antibacterial preparations is being increasingly introduced into clinical practice to treat bacterial infections caused by poly-resistant strains of microorganisms (Garimella et al., 2020). A necessary condition for combining antibacterial preparations is the rationality of their combination (Campos et al., 2020).

Antibiotic-resistant strains of microorganisms are a common cause of nosocomial infection; increase in the period of hospitalization of patients, costs for treatment and people's inability to work, increase in mortality rate (Barnes et al., 2018; Mobarki et al., 2018). In 2017, the report of EARSnet noted that the indicator of resistance of $E$. coli to cephalosporins of the III generation has reached the level of $14.9 \%$ in the European countries over recent decades (Annual report, 2018). Besides cephalosporins of the III generation, resistant isolates of $E$. coli are often resistant to antibiotics of classes of fluoroquinolones and aminoglycosides (Chervet et al., 2018).

Researchers have empirically and finally determined that combination therapy leads to better outcomes than monotherapy (Rodríguez-Baño et al., 2018). The importance of combining antibacterial preparations, in particular antibiotics and antiseptics, over the recent years is being pointed out by many scientists (Noites et al., 2014; Hansen et al., 2018). The synergic effect of applying such combinations was seen against a number of strains of multi-resistant Gram-negative bacteria (Tängdén, 2014; Thwaites et al., 2018; Schmid et al., 2019). Therefore, Garimella et al. (2018) performed a series of experiments on the effect of antibiotics: ampicillin, fosfomycin and ciprofloxacin both in isolated application and in combinations on the level of antibiotic-resistance of the clinical uropathogenic strain of Escherichia coli CFT073. The results of their study revealed that double and triple combinations of antibiotics significantly reduce antibiotic-resistance of $E$. coli of subpopulation CFT073. Djachenko et al. (2016) demonstrated that the greatest effect against PDR-strains of $P$. aeruginos $a$ and $E$. coli is exhibited by combination of fluoroquinolones and cephalosporins (in the study - ciprofloxacin and ceftazidime) For $76.9 \%$ of isolates of Pseudomonas aeruginosa and $66.7 \%$ of strains of enterobacteria, such combination had a synergic pattern.

The results of our study indicate that against a higher number of clinical isolates, synergic action of antibiotics (ceftriaxone, ofloxacin and gentamicin) and antiseptics (chlorhexidine, decasan) takes place during their use in combination, expressing through simultaneous 2-8-fold decrease in MIC of each of two tested preparations compared with their isolated application. The data we obtained substantiate results reported by other scientists according to whom the simultaneous use of chlorhexidine and gentamicin (or penicillin and tetracycline) against strains of $S$. aureus caused increase in susceptibility of the staphylococci to the corresponding antibiotic (Dopcea et al., 2020). The report by Fabry et al. (2014) described the synergic effect of antiseptic combined with erythromycin, doxycycline and linezolid against clinical isolates of $S$. aureus. 


\section{Conclusions}

In the study of the content isolated from wounds of surgery patients, we isolated 67 strains of conditionally pathogenic bacteria which were characterized by high level of resistance to antibiotics of various pharmacological groups. Eight isolates were identified to Staphylococcus aureus, 10 - to Staphylococcus epidermidis, 14 - Escherichia coli, 8 - Klebsiella pneumoniae, 8 - Proteus vulgaris, 7 - Proteus mirabilis, 12 - to Pseudomonas aeruginosa. The least effective antibiotics against the representatives of the Enterobacteriaceae family were tetracycline (resistance to which was displayed by $71.4 \%$ of isolates of $E$. coli, $75.5 \%$ of $K$. pneumoniae and all isolated cultures of Proteus) and penicillin preparations (the percentage of resistant tested isolates of enterobacteria exceeded $71.4 \%$ ). A total of $66.7 \%$ of cultures of $P$. aeruginosa was resistant to cefuroxime, ofloxacin and norfloxacin; $75.0 \%$ - to ceftriaxone and gentamicin; $83.3 \%$ - aminoglycoside antibiotics; $91.7 \%$ - to cefoxitin. All the isolated cultures of $P$. aeruginos $a$ were characterized by resistance to tetracycline and all the tested penicillin preparations. A total of $75.0 \%$ of strains of $S$. aureus was characterized by resistance to cefoxitin, ceftriaxone, ofloxacin, tetracycline, ampicillin and $62.5 \%$ - to ciprofloxacin, oxacillin and most aminoglycosides. A total of $60.0 \%$ of each clinical isolate of $S$. epidermidis was resistant to cefoxitin, cefotaxime, ciprofloxacin, tetracycline and ampicillin.

Among the antibiotic-resistant isolates, we found a number which exhibited resistance simultaneously to several antibiotics, therefore, according to the classification generally accepted in the medical practice, they were divided into the corresponding phenotypic groups: MDR-strains multidrug-resistant; XDR - extensive drug-resistant; PDR - pandrugresistant. The division of the cultures into the phenotypic groups according to the level of antibiotic-resistance allowed us to determine 4 poly-resistant isolates among the isolated strains of $S$. aureus, including two identified to MDR- and one to each of XDR- and PDR-strains; S. epidermidis - one XDR- and one MDR-isolate; K. pneumoniae - two MDR- and one XDR-isolate. Among eight isolated cultures of $P$. vulgaris, we found one pandrug-, one extensive drug- and one multidrug-resistant isolate, and among $P$. mirabilis - one extensive drug- and two multidrug-resistant strains. The highest number of poly-resistant isolates was found among E. coli and $P$. aeruginosa: three of each belonging to MDR-, two XDRand one of each to PDR-strains.

According to use of antibiotics (ceftriaxone, ofloxacin and gentamicin) and antiseptics (decasan and chlorhexidine) in the form of monopreparations against the tested cultures, we determined that minimum inhibitory concentration (MIC) of ceftriaxone, depending on the tested strain, ranged 64 to $256 \mu \mathrm{g} / \mathrm{mL}$; MIC of ofloxaciny $-1.0-64.0 \mu \mathrm{g} / \mathrm{mL}$, MIC of gentamicin $-1.0-128.0 \mu \mathrm{g} / \mathrm{mL}$, MIC of decasan -3.0 to $100.0 \mu \mathrm{g} / \mathrm{mL}$; MIC of chlorhexidine -4.0 to $250.0 \mu \mathrm{g} / \mathrm{mL}$. Against most clinical isolates, synergic action of antibiotics (ceftriaxone, ofloxacin and gentamicin) and antiseptics (chlorhexidine, decasany) took place while using them in combinations, expressing in simultaneous decrease in MIC of each of the tested preparations by 2-8 times, compared with their isolated application. Such positive effect of combined use of antibiotics and antiseptics opens broad prospects for treatment of wounds, surficial inflammatory processes, nosocomial infections caused by antibiotic-poly-resistant strains of microorganisms.

\section{References}

Agyekum, A., Fajardo-Lubián, A., Ai, X., Ginn, A., Zong, Z., Guo, X., Turnidge, J., Partridge, S., \& Iredell, J. (2016). Predictability of phenotype in relation to common-lactam resistance mechanisms in Escherichia coli and Klebsiella pneumonia. Journal of Clinical Microbiology, 54(5), 1243-1250.

Al-Talib, H., Alkhateeb, A., Syahriza, A., Ruzuk, A., Zulkifli, F., Hamizi, S., Muhammad, N., \& Karim, F. (2019). Effectiveness of commonly used antiseptics on bacteria causing nosocomial infections in tertiary hospital in Malaysia. African Journal of Microbiology Research, 13(10), 188-194.

Anesi, J., Lautenbach, E., Nachamkin, I., Garrigan, C., Bilker, W., Wheeler, M., Tolomeo, P., \& Han, J. (2016). Clinical and molecular characterization of community-onset urinary tract infections due to extended-spectrum cephalosporinresistant Enterobacteriaceae. Infection Control and Hospital Epidemiology, 37(12), 1433-1439.
Aslam, B., Wang, W., Arshad, M., Khurshid, M., Muzammil, S., Rasool, M., Nisar, M., Alvi, R., Aslam, M., Qamar, M., Salamat, M., \& Baloch, Z. (2018). Antibiotic resistance: A rundown of a global crisis. Infection and Drug Resistance, 11, $1645-1658$.

Barnes, M., Taracila, M, Rutter, J., Bethel, C., Galdadas, I., Hujer, A., Caselli, E., Prati, F., Dekker, P., Papp-Wallace, K., Haider, S., \& Bonomo, R. (2018). Deciphering the evolution of cephalosporin resistance to ceftolozane-tazobactam in Pseudomonas aeruginosa. MBio, 9(6), e2085-18.

Bassetti, M., Vena, A., Croxatto, A., Righi, E., \& Guery, B. (2018). How to manage Pseudomonas aeruginosa infections. Drugs in Context, 7, 1-18.

Botelho, J., Grosso, F., \& Peixe L. (2019). Antibiotic resistance in Pseudomonas aeruginosa - mechanisms, epidemiology and evolution. Drug Resistance Updates, 44, 26- 47.

Campbell, S., Goodnough, L., Bennett, C., \& Giori, N. (2018). Antiseptics commonly used in total joint arthroplasty interact and may form toxic products. The Journal of Arthroplasty, 33(3), 844-846.

Campos, M., Jimenez, F., Sanchez, G., Juarez, J., Morales, A., Canovas-Segura, B., \& Palacios, F. (2020). A methodology based on multiple criteria decision analysis for combining antibiotics in empirical therapy. Artificial Intelligence in $\mathrm{Me}-$ dicine, 102,101751

Chellat, M., Raguž, L., \& Riedl, R. (2016). Targeting antibiotic resistance. Angewandte Chemie International Edition, 55(23), 6600-6626.

Cheng, G., Ning, J., Ahmed, S., Huang, J., Ullah, R., An, B., Hao, H., Dai, M., Huang, L., Wang, X., \& Yuan, Z. (2019). Selection and dissemination of antimicrobial resistance in agri-food production. Antimicrobial Resistance and Infection Control, 158(8), 1-13.

Chervet, D., Lortholary, O., Zahar, J., Dufougeray, A., Pilmis, B., \& Partouche, H. (2018). Antimicrobial resistance in community-acquired urinary tract infections in Paris in 2015. Medecine at Maladies Infectieuses, 48(3), 188-192.

Dalhoff, A. (2012). Global fluoroquinolone resistance epidemiology and implictions for clinical use. Interdisciplinary Perspectives on Infectious Diseases, 2012, 976273.

Djachenko, V., Marjushhenko, A., Chigirins'ka, N., \& Kucaj, N. (2016). Efektivnist' diji kombinacij preparativ $\mathrm{z}$ grupy ftorhinoloniv $\mathrm{z}$ inshymy antibiotykamy na polirezystentni shtamy syn'ognijnoji palychky ta enterobakterij [Effectiveness of a combination of drugs from the group of fluoroquinolones with other antibiotics on multidrug-resistant strains of Pseudomonas aeruginosa and enterobacteria]. Biomedical and Biosocial Anthropology, 26, 71-73 (in Ukrainian).

Dopcea, N., Dopcea, I., Nanu, A., Diguta, C., \& Matei, F. (2020). Resistance and cross-resistance in Staphyloccocus sp. after prolonged exposure to different antiseptics. Journal of Global Antimicrobial Resistance, 21, 399-404.

European Centre for Disease Prevention and Control (2018). Surveillance of antimicrobial resistance in Europe. Annual report of the European Antimicrobial Resistance Surveillance Network (EARS-Net) 2017. ECDC, Stockholm.

Fabry, W., \& Kock, H.-J. (2014). In-vitro activity of polyhexanide alone and in combination with antibiotics against Staphylococcus aureus. Journal of Hospital Infection, 86(1), 68-72.

Garimella, N., Zere, T., Hartman, N., Gandhi, A., Bekele, A., Li, X., Stone, H., Sacks, L., \& Weaver, J. (2020). Effect of drug combinations on the kinetics of antibiotic resistance emergence in $E$. coli CFT073 using an in vitro hollow-fiber infection model. International Journal of Antimicrobial Agents, 55(4), 105861.

Hansen, S., Schwab, F., Zingg, W., \& Gastmeier, P. (2018). Process and outcome indicators for infection control and prevention in European acute care hospitals in 2011 to 2012 - Results of the Prohibit study. Eurosurveillance, 2(21), 1700513.

Jenull, S., Laggner, H., Hassl, I., Velimirov, B., Huettinger, B., \& Zemann, N. (2017). Cooperativity between antibiotics and antiseptics: Testing the bactericidal effect. Journal of Wound Care, 26(12), 720-726.

Kramer, A., Assadian, O., \& Koburger-Janssen, T. (2016). Antimicrobial efficacy of the combination of chlorhexidine digluconate and dexpanthenol. GMS Hygiene and Infection Control, 11, 1-6.

Magiorakos, A.-P., Srinivasan, A., Carey, R., Carmeli, Y., Falagas, M., Giske, C., Harbarth, S., Hindler, J., Kahlmeter, G., Olsson-Liljequist, B., Paterson, D., Rice, L., Stelling, J., Struelens, M., Vatopoulos, A., Weber, J., \& Monnet, D. (2012). Multidrug-resistant, extensively drug-resistant and pandrug-resistant bacteria: An international expert proposal for interim standard definitions for acquired resistance. Clinical Microbiology and Infection, 18(3), 268-281.

Marques, D., Machado, S., Ebinuma, V., Duarte, C., Converti, A., \& Porto, A. (2018). Production of $\beta$-lactamase inhibitors by Streptomyces species. Antibiotics, 7(3), 1-26.

Matthew, E., Lucy, J., Laura, C., \& Sutton, J. (2017). Mechanisms of increased resistance to chlorhexidine and cross-resistance to colistin following exposure of Klebsiella pneumoniae clinical isolates to chlorhexidine. Antimicrobial Agents and Chemotherapy, 61(1), 1-12.

Mobarki, N., Almerabi, B., \& Hattan, A. (2019). Antibiotic resistance crisis. International Journal of Medicine in Developing Countries, 3(6), 561-564.

Noites, R., Pina-Vaz, C., Rocha, R., Carvalho, M., Gonçalves, A., \& Pina-Vaz, I. (2014). Synergistic antimicrobial action of chlorhexidine and ozone in endodontic treatment. Journal of Biomedicine and Biotechnology, 2014, 1-6. 
Pachori, P., Gothalwal, R., \& Gandhi, P. (2019). Emergence of antibiotic resistance Pseudomonas aeruginosa in intensive care unit; A critical review. Genes and Diseases, 6, 109-119.

Palchykov, V. A., Zazharskyi, V. V., Brygadyrenko, V. V., Davydenko, P. O., Kulishenko, O. M., \& Borovik, I. V. (2020). Chemical composition and antibacterial effect of ethanolic extract of Buxus sempervirens on cryogenic strains of microorganisms in vitro. Chemical Data Collections, 25, 100323.

Pervical, S., Finnegan, S., Donelli, G., Vuotto, C., Rimmer, S., Benjamin, A., \& Lipsky, B. (2016). Antiseptics for treating infected wounds: Efficacy on biofilms and effect of pH. Critical Reviews in Microbiology, 42(2), 293-309.

Potapov, V., Vakulenko, E., \& Protasenko, Y. (2016). Vybor optimal'nyh antisepticheskih sredstv dlja obrabotki kostnoj polosti v processe hirurgicheskogo lechenija nagnoivshihsja radikuljamyh kist [The choice of optimal antiseptic agents for the treatment of the bone cavity in the process of surgical treatment of festering radicular cysts]. Ukrajinskyj Stomatologichnyj Al'manakh, 4, 40-42 (in Russian).

Rather, I., Byung-Chun K., Bajpai, V., \& Yong-HaPark (2017). Self-medication and antibiotic resistance: Crisis, current challenges, and prevention. Saudi Joumal of Biological Sciences, 24(4), 808-812.

Rodríguez-Baño, J., Gutiérrez-Gutiérrez, B., Machuca, I., \& Pascual, A. (2018). Treatment of infections caused by extended-spectrum-beta-lactamase-, AmpC-, and carbapenemase-producing Enterobacteriaceae. Clinical Microbiology Reviews, 31(2), e00079-17.

Schmid, A., Wolfensberger, A., Nemeth, J., Schreiber, P., Sax, H., \& Kuster, S. (2019). Monotherapy versus combination therapy for multidrug-resistant Gram-negative infections: Systematic review and meta-analysis. Scientific Reports, 9, 1-11.

Sweeney, M., Lubbers, B., Schwarz, S., \& Watts, J. (2018). Applying definitions for multidrug resistance, extensive drug resistance and pandrug resistance to clini- cally significant livestock and companion animal bacterial pathogens. The Journal of Antimicrobial Chemotherapy, 73(6), 1460-1463.

Tacconelli, E., Carrara, E., Savoldi, A., Harbarth, S., Mendelson, M., Monnet, D., Pulcini, C., Kahlmeter, G., Kluytmans, J., Carmeli, Y., Ouellette, M., Outterson, K., Patel, J., Cavaleri, M., Cox, E., Houchens, C., Grayson, M., Hansen, P., Singh, N., Theuretzbacher, U., \& Magrini, N. (2018). Discovery, research, and development of new antibiotics: The WHO priority list of antibiotic-resistant bacteria and tuberculosis. The Lancet Infectious Diseases, 18(3), 318-327.

Tängdén, T. (2014). Combination antibiotic therapy for multidrug-resistant Gram-negative bacteria. Upsala Joumal of Medical Sciences, 119(2), 149-153.

Thwaites, M., Hall, D., Stonebumer, A., Shinabarger, D., Serio, A., Krause, K. Marra, A., \& Pillar, C. (2018). Activity of plazomicin in combination with other antibiotics against multidrug-resistant Enterobacteriaceae. Diagnostic Microbiology and Infectious Disease, 92(4), 338-345.

Velez, R., \& Sloand, E. (2016). Combating antibiotic resistance, mitigating future threats and ongoing initiatives. Journal of Clinical Nursing, 25, 1886-1889.

Von Wintersdorff, C., Penders, J., van Niekerk, J., Mills, N., Majumder, S., van Alphen, L., Savelkoul, P., \& Wolffs, P. (2016). Dissemination of antimicrobial resistance in microbial ecosystems through horizontal gene transfer. Frontiers in Microbiology, 7, 1-10

Williamson, D., Carter, G., \& Howden, B. (2017). Current and emerging topical antibacterials and antiseptics: Agents, action, and resistance patterns. Clinical Microbiology Reviews, 30(3), 827-860.

Zazharskyi, V. V., Davydenko, P. O., Kulishenko, O. M., Borovik, I. V., \& Brygadyrenko, V. V. (2019). Antimicrobial activity of 50 plant extracts. Biosystems Diversity, 27(2), 163-169. 